\title{
Diseño y construcción de prototipo de máquina deshidratadora y pulverizadora de cebolla
}

\section{Design and construction of dehydrator and pulverizer prototype machine for onion.}

\author{
Luis Enrique Arteaga Noguera \\ Programa de Ingeniería Mecánica, Director grupo de investigación INMECNAR, Corporación Universitaria Autónoma de \\ Nariño, Pasto, Colombia \\ luis.arteagaeaunar.edu.co
}

\begin{abstract}
Resumen - En el siguiente artículo presenta una propuesta para la industrialización de la cebolla cabezona, mediante el diseño y construcción el prototipo para deshidratar y pulverizar cebolla. Se describen las pruebas realizadas, las cuales incluyen las curvas de secado y pruebas de granulometría del producto (ASTM C136-05), y los diferentes aspectos que se tuvieron en cuenta para el diseño de la máquina.
\end{abstract}

Palabras clave- Diseño de máquinas, deshidratadora, pulverizadora, cebolla.

Abstract- The following article presents a proposal for the industrialization of the onion, through design and construction of dehydrated and pulverize prototype of onion. Describes the tests performed, which include the drying curves and for sieve analysis of the product (ASTM C136-05), and the different aspects that were taken into account in the design of the machine.

Key Word -Machine Design, dehydrator, pulverizer, onion.

\section{INTRODUCCIÓN}

La cebolla cabezona ocupa el segundo lugar dentro de los productos alimenticios con mayor volumen de importación a nivel mundial. El flujo de importación se dirige fundamentalmente hacia Asia, con el 42\%, sigue Europa con el 39\% y América con el $14 \%$. La Tendencia apunta a que las compras externas podrían llegar a los 8 millones de toneladas [1].

En Colombia, el Programa de Transformación Productiva del Ministerio de Comercio, Industria y Turismo, priorizó la cebolla como una de las hortalizas con mayor potencial de exportación. Este producto se exporta procesado en conserva o deshidratada (incluye pulverizada) [2].

En el Departamento de Nariño, la cebolla es uno de los productos hortifruticolas que más se destacan de la subregión Pasto, junto con el frijol, maíz, papa, tomate de árbol, tomate de mesa, lulo, cítricos, arveja, yuca, plátano, piña y mora, fresa y uchuva. La subregión Pasto, con respecto al total de la producción en toneladas que se producen semestralmente en el departamento, aporta cebolla en un $70.1 \%$. [3].
Los municipios que se dedican al cultivo de la cebolla cabezona son: Iles, Imués, Funes, Yacuanquer, Samaniego, el Tambo, el Contadero y el Peñol. Durante la época de cosecha, se presenta una sobre oferta del producto; lo cual ocasiona un bajo precio de compra, representando pérdidas para el productor. Por ésta razón, un porcentaje considerable de la cebolla es desechado o guardado, exponiéndolo a su degradación o pudrición El diseño de una máquina deshidratadora y pulverizadora de cebolla aporta a la solución de este problema, al permitir conservar el producto, disminuyendo el desperdicio y generando valor agregado [4].

\section{PRODUCCIÓN DE CEBOLLA EN NARIÑO}

La Tabla 1, muestra la producción de cebolla fresca en el departamento de Nariño para el primer semestre del año 2012, con un total de 246 Ha sembradas y una producción de 2296.4 toneladas semestrales [5]. Se estima que el 5\% de la producción total se desperdicia en tiempo de poscosecha [6].

\begin{tabular}{l|cc} 
Municipio & $\begin{array}{c}\text { Área sembrada } \\
\text { (Has) }\end{array}$ & $\begin{array}{c}\text { Producción } \\
\text { (Ton) }\end{array}$ \\
\hline Contadero & 2.0 & 42.4 \\
El peñol & 10.0 & 140.0 \\
El Tambo & 10.0 & 80.0 \\
Funes & 50.0 & 750.0 \\
Iles & 57.0 & 684.0 \\
Imues & 70.0 & 280.0 \\
Samaniego & 2.0 & 24.0 \\
Taminango & 10.0 & 104.0 \\
Tangua & 20.0 & 27.0 \\
Yacuanquer & 15.0 & 165.0 \\
Total & 246.0 & 2296.4
\end{tabular}

Tabla 1. Producción de cebolla en Nariño (Primer semestre de 2012)

La Figura 1, indica el comportamiento de la producción de cebolla cabezona en el Departamento de Nariño. Estos datos fueron recopilados de los Consolidados Agropecuarios de Nariño en el periodo 2007 a 2012. Se puede apreciar que existe una tendencia creciente de la producción, con un valor promedio de crecimiento de 160 toneladas anuales [5], [7], [8], [9], [10], [11]. 


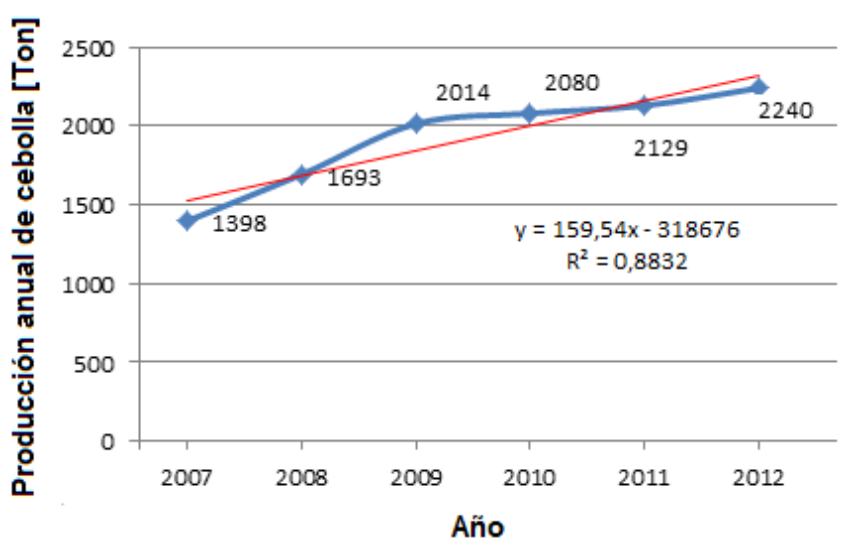

Figura 1. Producción de cebolla en Nariño (2007 - 2012).

\section{CONDICIONES DE CALIDAD DEL PRODUCTO}

El mercado de productos alimenticios es muy exigente en cuanto a las normas de calidad, El producto procesado debe cumplir con las normas especificadas en la Tabla 2.

\begin{tabular}{ll}
\hline Características & Condiciones \\
\hline Humedad máxima & $6 \%$ \\
Cenizas & $5 \%$ \\
Organolépticas & El producto debe tener color, olor y sabor \\
& característicos de la cebolla fresca \\
& después de mantenerse durante 10 \\
& minutos a $5{ }^{\circ} \mathrm{C}$. El color debe ser blanco, \\
& no tostado. \\
Aditivos & Exenta de productos diluyentes tales \\
& como: harina, almidón, y materiales \\
& minerales. \\
Impurezas & Libre de pelos de roedores y de \\
& fragmentos de insectos. \\
- Bacterias & 10.000 colonias/gramo \\
- Hongos & 50 colonias/gramo \\
- Levaduras & 50 colonias/gramo \\
Granulometría & $100 \%$ malla 80 \\
\hline
\end{tabular}

Tabla 2. Normas de calidad para la cebolla deshidratada [12].

Las condiciones de humedad, color y sabor, están influenciadas por el proceso de secado, específicamente por la temperatura y el tiempo de permanencia del producto en el horno, para lo cual se deben realizar diferentes pruebas para obtener las curvas de secado del producto.

Para cumplir con los requerimientos sanitarios, es necesario realizar un control estricto de la planta y del proceso, para garantizar las condiciones básicas de higiene en la fabricación de alimentos, tal como se establece en el Artículo $5^{\circ}$. Buenas Prácticas de Manufactura, promulgado por el Ministro de Salud y Protección Social, RESOLUCIÓN 2674 DE 2013. Adicional al cumplimiento de estas condiciones, es necesario tramitar el registro sanitario ante el Instituto Nacional de Vigilancia de Medicamentos y Alimentos (Invima). [13] Con el fin de determinar el tamaño del grano de la cebolla en polvo, se realizan pruebas de acuerdo con la norma ASTM C 136 (Método de Ensayo Normalizado para la Determinación
Granulométrica de Agregados Finos y Gruesos) [14]. Para la cebolla deshidratada se verifica que el producto sea homogéneo, con una granulometría Tipo I (en polvo) donde el $2 \%$ queda retenido en una malla 45 , el $25 \%$ en una malla 80 y el $30 \%$ en una malla 100.

\section{DESCRIPCIÓN DEL PROCESO}

La Figura 2, muestra la descripción total del proceso de fabricación de cebolla en polvo. La máquina diseñada se encarga únicamente de los procesos de deshidratación y molienda.

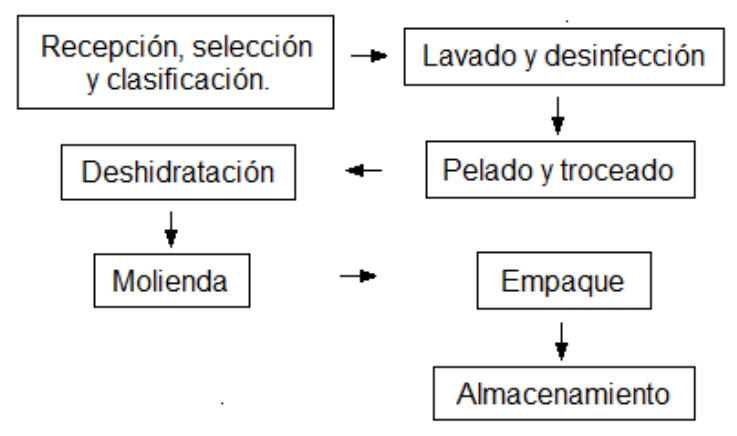

Figura 2. Proceso de fabricación cebolla deshidratada

A. Condiciones de entrada del producto.

Previo al proceso de secado, es necesario preparar la cebolla de forma manual. Para ello, se inicia con el proceso de recepción, selección y clasificación de la materia prima. Se recomienda escoger los bulbos de la cebolla de buena calidad, que reúnan los siguientes requisitos: sanos, libres de daños mecánicos causados por plagas y enfermedades, bien cubiertos por capas externas o túnicas, bien secos y homogéneos [15].

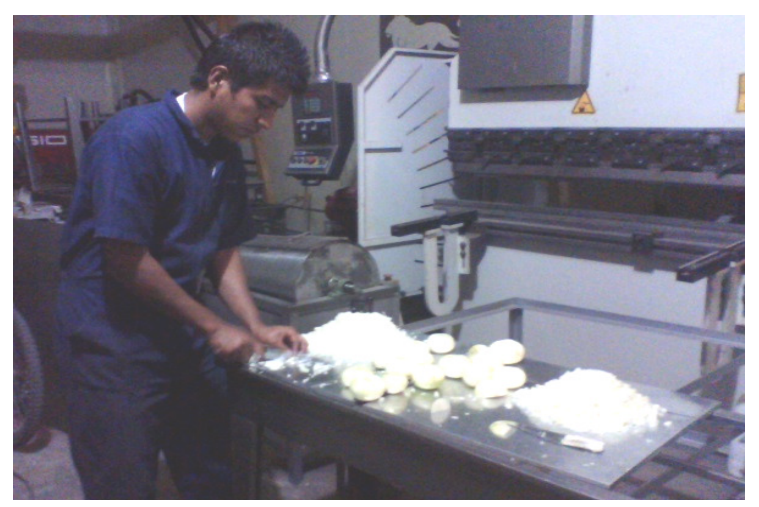

Figura 3. Tratamiento previo del producto

Posteriormente, se efectúa el lavado de desinfección de la cebolla. A continuación se realiza el proceso de pelado, en el cual se separa la parte exterior o cáscara de la hortaliza, dejando la parte blanca. Finalmente, se efectúa el lavado y troceado de la cebolla en pedazos. El corte se realiza en el 
sentido vertical al eje de la cebolla, hasta obtener trozos de un tamaño aproximado de $5 \times 10 \times 2 \mathrm{~mm}$, con el fin de facilitar el proceso de deshidratación.

\section{Proceso de deshidratación:}

El proceso de deshidratación se realiza en un horno rotatorio, a una temperatura entre 50 y $60{ }^{\circ} \mathrm{C}$, con el fin de conservar las características de olor y sabor del producto.

\section{Proceso de molienda:}

El proceso de molienda es una operación mecánica que trasforma la materia sin alterar su naturaleza o propiedades físicas del producto. Este proceso se realiza empleando un molino de martillos.

\section{Proceso de empaque y almacenamiento:}

La cebolla en polvo debe almacenarse sobre plataformas de madera o superficies elevadas del piso, para protegerlo de la humedad, el derrame de líquidos y las suciedades, en bodegas cubiertas, en ambientes secos, con buena ventilación y a temperatura ambiente. [12]

En las bodegas de almacenamiento se debe contar con un plan integral de control de plagas, limpieza y buenas prácticas de manufactura. Una vez se abra el empaque, para emplear una parte, se debe cerrar inmediatamente para evitar la exposición a la humedad del ambiente y a la contaminación microbiana.

\section{B. Cálculos}

Para el diseño de la máquina se partió de la cantidad de producto a procesar. A partir de éste valor se procedió a calcular la deshidratadora y el molino de martillos. Los cálculos realizados se resumen a continuación:

\section{Cantidad de producto a procesar}

2. Deshidratadora

- Volumen de cebolla a deshidratar

- Cantidad de agua a remover

- Dimensiones del tambor deshidratador

- Velocidad de rotación del tambor

- Cálculo y selección del ventilador

- Cálculo y selección del quemador

- Eficiencia de secado

- Potencia necesaria para el tambor deshidratador

3. Molino de martillos

- Capacidad de molienda

- Tiempo de molienda

- Velocidad de rotación

- Diseño de martillos

- Potencia necesaria para el molino

\section{Sistema de potencia}

- Selección del motor para el tambor deshidratador y el molino de martillos.

- Diseño transmisión

- Diseño y selección de elementos mecánicos (ejes, rodamientos, tornillos, etc.)

5. Sistema eléctrico y de protección

C. Cantidad de producto a procesar

La cantidad de producto a deshidratar se estima a partir de los datos recopilados en sobre la producción de cebolla en el Departamento de Nariño. La máquina deshidratadora se ubica en Pasto, razón por la cual se toma el $70.1 \%$ de la producción total del departamento. De este total, se toma el $4 \%$, que representa la cebolla que se pierde en poscosecha y que puede ser aprovechada para su venta en polvo. A éste valor se lo divide entre 6 , para obtener la producción mensual promedio de cebolla a procesar.

$2296.4\left(\frac{\text { Ton }}{\text { Sem }}\right)\left(\frac{1 \text { Sem }}{6 \text { meses }}\right) * 70.1 \% * 4 \%=10,6$ Ton $/$ mes

Para estimar la cantidad de cebolla en kilogramos que la máquina debe procesar es necesario considerar el tiempo de secado. Se espera que la máquina pueda deshidratar y pulverizar la cebolla en un solo turno de trabajo. La máquina trabajará en tres turnos de 8 horas. Para obtener el valor en kilogramos, se multiplica por 1000 y se divide éste valor entre 24 días laborables al mes en tres turnos de trabajo.

$$
10,6\left(\frac{\text { Ton }}{\text { mes }}\right)\left(\frac{1000 \mathrm{~kg}}{1 \text { Ton }}\right)\left(\frac{1 \text { mes }}{24 \text { dias }}\right)\left(\frac{1 \text { dia }}{3 \text { turnos }}\right) \square 147 \frac{\mathrm{kg}}{\text { turno }}
$$

Los resultados del proceso de cálculo se resumen en la Tabla 3 , donde se muestran las especificaciones de la máquina.

\begin{tabular}{lc}
\hline Capacidad de producción & $\mathbf{1 4 7} \mathrm{kg} /$ turno \\
\hline Cantidad de agua a remover & $120.23 \mathrm{~kg}$ \\
Dimensiones del & $\mathrm{h}=0.8 \mathrm{~m}$ \\
tambor deshidratador & $\mathrm{d}=0.76 \mathrm{~m}$ \\
Velocidad angular del tambor & $10 \mathrm{rpm}$ \\
Tiempo de secado & $7 \mathrm{horas}$ \\
Ventilador axial monofásico de & $\mathrm{Q}=0.3 \mathrm{~m}^{3} / \mathrm{s}$ \\
6" (110 V AC). & $\mathrm{P}=0.25 \mathrm{watt}$ \\
Quemador a gas & $10.000 \mathrm{BTU} 7 \mathrm{~h}$ \\
Eficiencia de secador & $10 \%$ \\
Potencia tambor & $0.112 \mathrm{hp}$ \\
Tiempo de molienda & $20 \mathrm{~min}$ \\
Velocidad angular martillo & $3272 \mathrm{rpm}$ \\
Motor eléctrico (110 V AC). & $0.5 \mathrm{hp} / 1750 \mathrm{rpm}$ \\
Transmisión unidad de secado & Cadena paso $1 / 4$ ". \\
Transmisión unidad de molido & Banda $3 \mathrm{~V}$ \\
\hline
\end{tabular}

Tabla 3. Especificaciones de la máquina. 


\section{Procesos experimentales}

1. Volumen de cebolla a deshidratar: Este cálculo se realiza de manera experimental, a partir de la medición de cebolla picada. Se emplean trozos de un tamaño aproximado de 10 × 5 × $2 \mathrm{~mm}$. Como resultado de las pruebas, para una masa de 220 gramos $(0.22 \mathrm{~kg})$, se obtiene un volumen promedio de $550 \mathrm{ml}\left(5.5 \times 10^{-4} \mathrm{~m}^{3}\right)$. El volumen de $70 \mathrm{~kg}$ de cebolla se determina a partir de:

$$
147 \mathrm{~kg}\left(\frac{5.5 \times 10^{-4} \mathrm{~m}^{3}}{0.22 \mathrm{~kg}}\right)=0.368 \mathrm{~m}^{3}
$$

2. Dimensiones del tambor deshidratador: El volumen del tambor debe garantizar el movimiento y flujo de aire caliente dentro de la cámara, por lo cual se recomienda aumentar su tamaño en un $25 \%$.

$$
V_{t}=0.368 m^{3}+\left(0.368 m^{3} \times 25 \%\right)=0.46 m^{3}
$$

El radio del tambor se calcula a partir del volumen de un cilindro. Se determina una longitud $h=0.8 \mathrm{~m}$.

$$
r=\sqrt{\frac{V_{t}}{\pi * h}}=\sqrt{\frac{0.368 m^{3}}{\pi(0.8 m)}}=0.38 \mathrm{~m}
$$

3. Curvas de secado:Para determinar las medidas exactas del prototipo se deben tener en cuenta las curvas de secado de los alimentos, las cuales se obtienen a partir de datos experimentales. Estas curvas permitirán demostrar la efectividad del sistema de tambor diseñado. Por ésta razón, se efectuaron dos pruebas. La primera en lecho estático y la segunda empleando un tambor rotatorio. Estas pruebas se diseñaron teniendo en cuenta variables como: velocidad del aire, temperatura, humedad, presión, cantidad y tamaño del producto a procesar. Se empleó un secador con resistencias eléctricas y un ventilador de $60 \mathrm{~Hz}, 110 \mathrm{~V}, 0.76 \mathrm{KW}$. Se midió la humedad de la cebolla antes de la prueba para verificar que se encuentre el 12 y $14 \%$.

Prueba 1: En la primera prueba se utiliza cebolla en trozos de 5 × 0.8 × $1 \mathrm{~mm}$, en un lecho estático. Las mediciones se realizaron cada 20 minutos.

\begin{tabular}{lc}
\hline Peso antes de iniciar la prueba & $\mathbf{1 1 9 . 8}$ gramos \\
\hline Peso de sólido seco (ws) & 8.3 gramos \\
Porcentaje de sólido seco (\%ss) & $0.08 \%$ \\
Humedad de equilibrio (Xlibre) & 0.0002 \\
Volumen del producto & $4 \mathrm{~mm}^{3}$ \\
Diámetro lecho estático & $13.9 \mathrm{~cm}$ \\
\hline
\end{tabular}

Tabla 4. Datos de secado (Prueba 1)

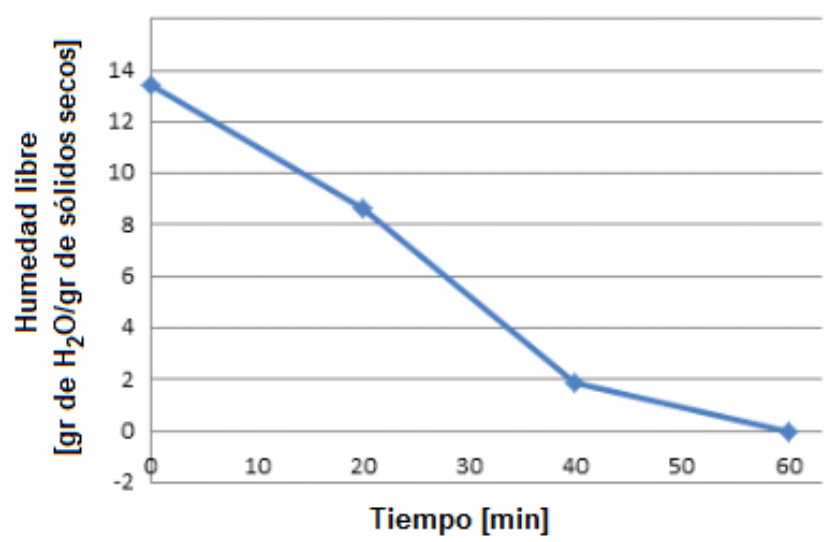

Figura 4. Humedad libre vs tiempo de secado (Prueba 1)

En esta gráfica se aprecia un descenso de la humedad a medida que aumenta el tiempo en el secador. Sin embargo, las caídas bruscas en la pendiente indican tramos donde se produce una variación de la velocidad de secado.

Prueba 2: En la segunda prueba se utiliza cebolla en trozos más grandes, de $5 \times 2 \times 10 \mathrm{~mm}$, en un tambor rotatorio de malla metálica. Las mediciones se realizaron cada 20 minutos.

\begin{tabular}{lc}
\hline Peso antes de iniciar la prueba & $\mathbf{2 1 4 . 4}$ gramos \\
\hline Peso de sólido seco (ws) & 17.15 gramos \\
Porcentaje de sólido seco (\%ss) & $0.08 \%$ \\
Humedad de equilibrio (Xlibre) & 0.0002 \\
Volumen del producto & $4 \mathrm{~mm}^{3}$ \\
Volumen del tambor & $3004.14 \mathrm{~cm}^{3}$ \\
\hline
\end{tabular}

Tabla 5. Datos de secado (Prueba 2)

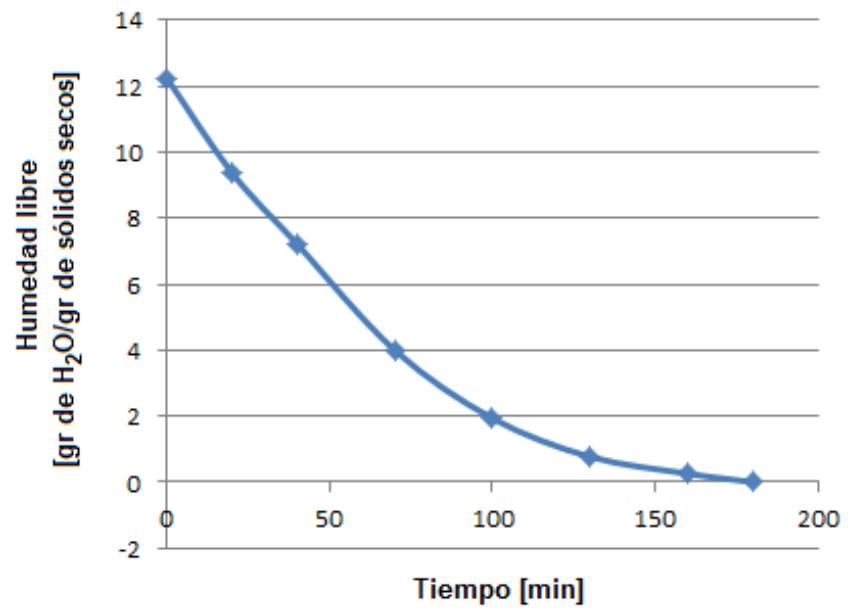

Figura 5. Humedad libre vs tiempo de secado (Prueba 2)

En ésta se aprecia un cambio progresivo de la pendiente de la curva de secado, lo cual indica una velocidad aproximadamente constante de secado. A partir del análisis de éstas gráficas se pudo verificar la ventaja del sistema de tambor giratorio que se empleará en el diseño de la máquina. 
4. Energía de triturado: Mediante ésta prueba se determinó la energía necesaria para triturar los granos de cebolla seca $(E$ ruptura $)$. Para ello, se dejó caer un martillo metálico de 20 gramos $(m)$ a diferentes alturas, sombre una masa de cebolla seca de 0.011 gramos con una humedad entre el 12 y $14 \%$. La altura $(h)$ estimada para obtener el tamaño de grano deseado fue de $20 \mathrm{~cm}$. La energía se calcula la energía mediante la ecuación.

$$
E_{\text {ruptura }}=m g h=0.020 \mathrm{~kg} * 9.81 \frac{\mathrm{m}}{\mathrm{s}^{2}} * 0.2 \mathrm{~m}=0.04 \mathrm{~N} . \mathrm{m}
$$

5. Pruebas de granulometría: Con el fin de determinar el tamaño del grano de la cebolla en polvo, se realizaron pruebas de acuerdo con la norma ASTM C 136. En esta prueba se utiliza una muestra de ensayo de agregado seco de masa conocida, la cual se separa a través de una serie de mallas de aberturas progresivamente más pequeñas; para determinar la distribución por tamaño de partículas. Los porcentajes se calculan a partir del peso de agregado que queda en las diferentes mallas, para lo cual se hizo uso de una báscula calibrada.

Para la cebolla deshidratada se verificó la homogeneidad del producto, con una granulometría Tipo I (en polvo) donde el $2 \%$ del producto queda retenido en una malla 45 , el $25 \%$ en una malla 80 y el $30 \%$ en una malla 100 .

\section{E. Descripción de la máquina}

La máquina deshidratadora y pulverizadora de cebolla dispone de un tablero para el control de los procesos. Este tablero permite visualizar y controlar la temperatura de secado, por medio de un pirómetro digital. El flujo de gas es controlado por medio de una electroválvula. Así mismo, cuenta con un interruptor para el quemador el cual activa un encendedor de chispa eléctrica. El sistema de potencia utiliza contactores y una protección térmica para el motor.

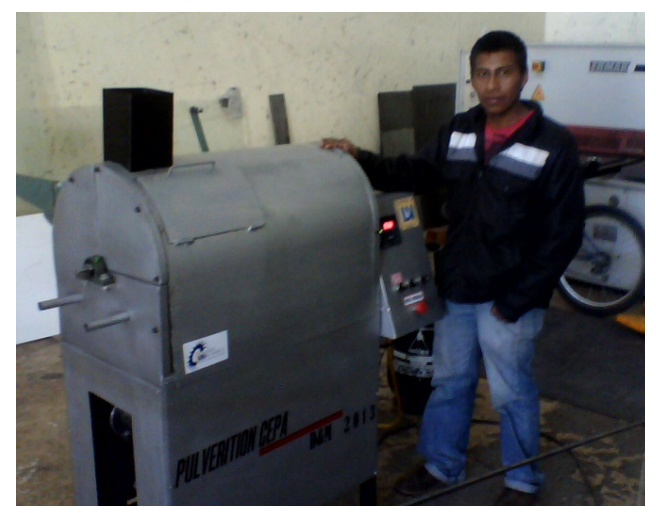

Figura 6. Prototipo de máquina deshidratadora y pulverizadora.

El sistema de secado utiliza un tambor rotatorio impulsado por un motor eléctrico de $1 / 2 \mathrm{hp}$, que emplea una trasmisión por cadena para permitir el movimiento del tambor deshidratador.

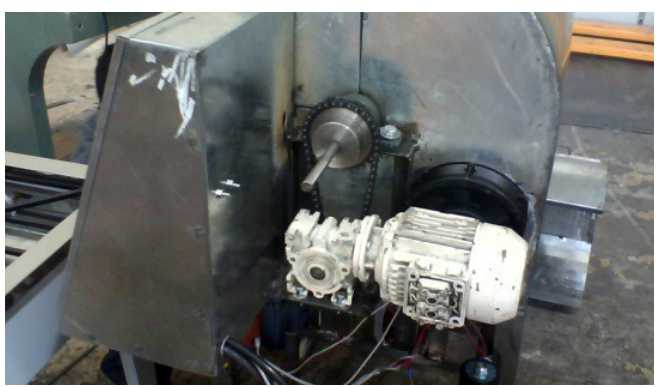

Figura 7. Transmisión unidad de deshidratado.

El tambor de secado es calentado en su superficie por quemadores alimentados por gas, transmitiendo el calor por contacto, mientras una corriente de aire circula internamente. Para ello, se emplea un ventilador que hace circular aire, produciendo un lecho de aire caliente uniforme.

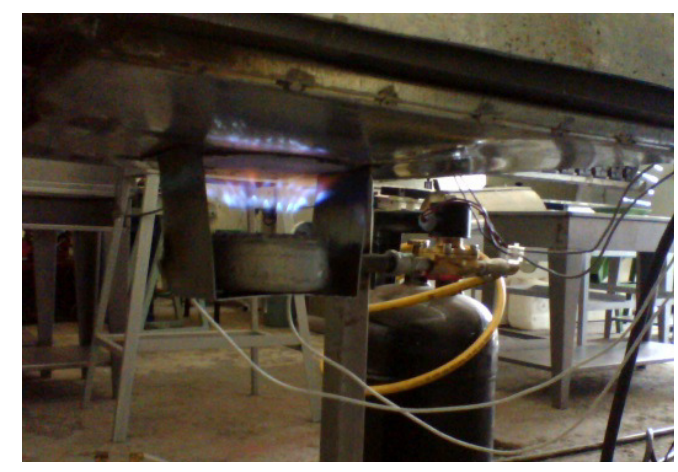

Figura 8. Calentamiento por gas

Antes de iniciar con el proceso de molido, es necesario abrir la escotilla para permitir el paso por gravedad de la materia prima hacia los martillos. A continuación se da inicio al proceso por medio del interruptor de molido. El proceso de molienda utiliza molinos de martillo, los cuales se usan ampliamente para triturar productos fibrosos, pegajosos o cristalinos. Este equipo es usado como complemento en la molienda de productos deshidratados para la elaboración de harinas y productos granulados en la industria alimenticia. El molino de martillos utiliza un sistema de trasmisión por banda. Es necesario lograr un correcto balanceo del eje con los martillos con el fin de minimizar las vibraciones y los ruidos.

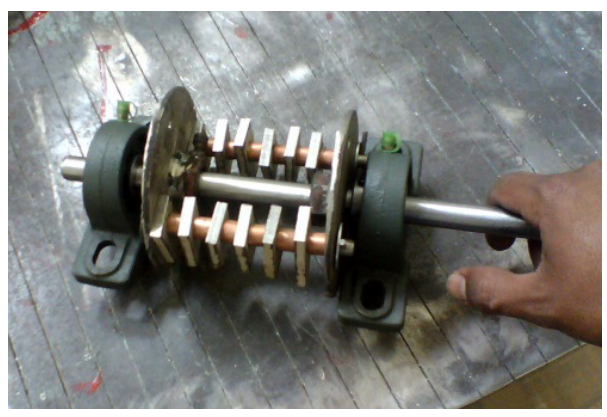

Figura 9. Martillos. 


\section{CONCLUSIONES}

- En el Departamento de Nariño, los municipios del Contadero, El peñol, El Tambo, Funes, Iles, Imues, Samaniego, Taminango, Tangua y Yacuanquer, presentan una considerable producción de cebolla cabezona, la cual se desperdicia en tiempo de cosecha; por efecto de la disminución del precio.

- La implementación de una máquina deshidratadora y pulverizadora de cebolla, permite preservar y generar valor agregado a la cebolla que no puede venderse fresca.

- A partir del análisis de las curvas de secado se pudo comprobar que la implementación de un sistema rotativo garantiza la obtención de un producto homogéneo, y disminuye los costos y tiempo del proceso.

- En el proceso de pulverizado, se pudo evidenciar la efectividad del molino de martillos para productos secos.

- Se estableció una energía promedio de triturado igual a 0.04 N.m, para muestras de 0.011 gr con una humedad relativa entre el 12 y $14 \%$.

- Se verificó el adecuado movimiento y flujo de aire en la cámara de secado, con un tamaño de tambor superior en un $25 \%$ del volumen procesado.

- El tamaño de la cebolla picada al inicio del proceso de deshidratación, influye en el comportamiento de las curvas de secado y en la calidad final del producto.

\section{RECOMENDACIONES}

- Aunque la máquina fue diseñada para deshidratar y pulverizar cebolla, puede utilizarse para realizar las mismas operaciones con otras tipos hortalizas, como el ajo. En este caso, es necesario realizar las curvas de secado para poder identificar las condiciones de operación que garanticen la calidad del producto.

- Durante el proceso de construcción de la máquina, es necesario tener en cuenta las normas de seguridad con el fin de evitar accidentes y lesiones personales.

- A pesar de que la máquina se diseñó cumpliendo las normas de buenas prácticas de manufactura, es necesario verificar los conteos de bacterias hongos y levaduras mediante pruebas de laboratorio.

\section{REFERENCIAS}

[1] DANE. "Boletín Semanal Precios Mayoristas. No. 40". Frutas y hortalizas tipo exportación. Marzo 2013. Disponible

en: https://www.dane.gov.co/files/investigaciones/agropecua rio/sipsa/Semana_16mar_22mar_2013.pdf

[2] Ministerio de Agricultura y Desarrollo Rural, "Sistema de inteligencia de mercados". Cebolla procesada. Disponible

en: http://www.agronet.gov.co/www/docs_agronet/2006719 105355_CEBOLLaPROCESADAMayo.pdf
[3] Alcaldía de Pasto, "Descripción de las actividades agropecuarias con importancia económica de la región". Disponible

en: https://www.google.com.co/url?sa=t\&rct=j\&q=\&esrc=s $\&$ source $=$ web $\&$ cd $=1 \&$ cad $=$ rja $\&$ uact $=8 \&$ ved $=0 \mathrm{CBoQFj}$

AA\&url=http $\% 3 \mathrm{~A} \% 2 \mathrm{~F} \% 2 \mathrm{Fwww}$.pasto.gov.co\%2Findex .php $\% 2$ Fcomponent $\% 2$ Fphocadownload $\% 2$ Fcategory $\% 2$ F279-

epsagro\%3Fdownload\%3D5798\%3Aentregable_2_2014 \&ei=8r_0U6iCGoW2ogS124AQ\&usg=AFQjCNGzeDtc0QAr-

YDxYdplQeMBa6S3w\&sig2=WXoHa2yLXrcpOcgauJc NUg

[4] J. Nandar, M. Micanquer. "Diseño y construcción de un prototipo de máquina deshidratadora y pulverizadora de cebolla". San Juan de Pasto. 2013. Trabajo de grado Ingeniero Mecánico. Corporación Universitaria Autónoma de Nariño, Facultad de Ingeniería.

[5] Gobernación de Nariño. "Consolidado Agropecuario Nariño 2012".

[6] G. Cifuentes. "Estudio sobre la aplicación de buenas prácticas agrícolas en cebolla cabezona y tomate de mesa". Tunja, 2009. UNAD. Observatorio de cadenas productivas.

[7] Gobernación de Nariño. "Consolidado Agropecuario Nariño 2011".

[7] Gobernación de Nariño. "Consolidado Agropecuario Nariño 2010".

[8] Gobernación de Nariño. "Consolidado Agropecuario Nariño 2009".

[9] Gobernación de Nariño. "Consolidado Agropecuario Nariño 2008".

[10] Gobernación de Nariño. "Consolidado Agropecuario Nariño 2007".

[11] Ministro de Salud y Protección Social, "Resolución 2674 DE 2013", Por la cual se reglamenta el artículo 126 del Decreto-ley 019 de 2012 y se dictan otras disposiciones. Disponible en: http://www.alcaldiabogota.gov.co/sisjur/normas/Norma1 .jsp?i=54030

[12] ALITECNO. "Cebolla en polvo EXT 7814". Disponible en: $\quad$ http://www.alitecnoperu.com/industrias/ho-reca/item/cebolla-en-polvo-ext-7814

[13] ASTM. "Standard Test Method for Sieve Analysis of Fine and Coarse Aggregates". Standard ASTM C136, 2006.

[14] NTC. "Frutas frescas. Cebolla cabezona. Almacenamiento y transporte" Estándar NTC 1221-3, Junio 1996.

[15] NORTON, Robert. Diseño de máquinas, México. Prentice Hall, 1999.

[16] MOTT, Robert L., et al. Diseño de elementos de máquinas. Pearson Educación, 2006. 